\title{
Anesthetic management of a case of umbilical cord prolapse
}

\author{
Lisa Borretta BSc ${ }^{1}$, Ana Sjaus MD, FRCPC 2 \\ 'Class of 2017, Faculty of Medicine, Dalhousie University \\ ${ }^{2}$ Department of Women's and Obstetric Anesthesia, Dalhousie University
}

\begin{abstract}
There are no recent case reports published in the literature detailing the anesthetic management of a case of umbilical cord prolapse (UCP). We describe the successful anesthetic management of a 36-year-old multiparous patient who was rushed to the operating room for a cesarean section following the diagnosis of UCP immediately after a controlled amniotomy for arrest of cervical progression. Amniotomies are common and safe procedures done in order to augment labour, of which cord prolapse is a rare complication. Immediate delivery is the optimal management.
\end{abstract}

$\mathrm{U}$ mbilical cord prolapse (UCP) is a well-known obstetrical emergency in which the umbilical cord descends out of the uterus and through the cervix, either in advance (overt prolapse) or alongside (occult prolapse) the fetal presenting part. UCP is a rare obstetrical emergency, occurring in approximately $0.14-0.62 \%$ of deliveries. ${ }^{1}$ Of major concern in this emergency situation is that the prolapse can result in cord compression or vasospasm and compromise blood flow to the fetus, which may result in perinatal mortality or compromise. ${ }^{2}$ While the incidence of cord prolapse has not substantially changed over the past century, the outcomes have considerably improved from $32-47 \%$ fetal mortality in the 1950 s to less than $10 \%$ over the past two decades. This is due to enhanced neonatal resuscitation, improved care, and more frequent cesarean deliveries..$^{3-6}$

Risk factors associated with cord prolapse include: multiparity, malpresentation, prolonged labour, prematurity, pelvic deformities, low birth weight, polyhydramnios, and artificial rupture of membranes (AROM)., ${ }^{4,6-8}$ Despite these risk factors, a recent study found that UCP most commonly occurs in low-risk women at term. ${ }^{7}$ It has been suggested that approximately half the cases of cord prolapse are due to iatrogenic causes. ${ }^{9}$ However, iatrogenic cord prolapse has not been shown to result in poor outcomes due to continuous fetal monitoring and the availability of appropriate interventions in a hospital setting. In addition, there may be iatrogenic cord prolapse in a case where risk factors were present and may have resulted in spontaneous cord prolapse otherwise. ${ }^{9-11}$

UCP is diagnosed clinically by the presence of a palpable or visible cord and is most often accompanied by severe, rapid fetal heart rate decelerations. In situations where fetal bradycardia is present after the rupture of membranes, UCP must be ruled out. As the degree of cervical dilatation is typically not amenable to immediate vaginal delivery, a cesarean section is most commonly the optimal mode of delivery following the diagnosis of an UCP. In a scenario where a vaginal delivery is predicted to be safer and quicker, the decision to move forward is based on fetal heart tracing. ${ }^{9}$ Indeed, a recent large-scale retrospective cohort study concluded that vaginal delivery is preferred when possible, as it has been associated with a lower risk of fetal injury. ${ }^{7}$

We report a case of successful anesthetic management of an emergency cesarean section to treat a case of UCP.

\section{Case presentation}

A 36-year old G3P1 female was admitted at 37 weeks and six days gestation for induction of labour for gestational diabetes with decreasing insulin requirements. She had an epidural catheter in place that was administered 12 hours previously. Her pregnancy was otherwise uncomplicated. Her past medical history was significant for migraines, uterine fibroids, and depression. Past obstetrical history included a spontaneous vaginal delivery three years previous of a live female infant at 37 weeks gestation. Her medications included pre-natal vitamins, ranitidine, insulin NPH (36 units at bedtime) and insulin aspart (12 units at breakfast, 8 units at lunch and 12 units at dinner). Oxytocin protocol was started and the maximum infusion rate was reached. At this time, an AROM was deferred, as the head was not well applied. She continued to receive the maximum rate of oxytocin, with multiple cervical checks continuing to be unfavourable for an AROM. However, after 14 hours of maximum oxytocin without any cervical progression for 3.5 hours, the patient consented to a controlled AROM. Immediately after, UCP was diagnosed and she was rushed to the operating room (OR). 
On examination, the patient was $112 \mathrm{~kg}$, had a Mallampati Class I airway, thyromental distance of $6 \mathrm{~cm}$, mouth opening of four $\mathrm{cm}$, and full range of cervical mobility. Fetal heart rate monitor displayed persistent bradycardia between $80-90$ beats per minute (BPM) upon arrival to the OR.

As her airway was favourable for orotracheal intubation, a rapid sequence induction was performed with cricoid pressure and preoxygenation. The intubation was smooth and general anesthetic required four minutes to perform from entry to the OR until readiness for surgery. Standard anesthetic monitoring was continued until extubation.

Upon entry to the OR, the patient was given a top-up of lidocaine $2 \%$ with epinephrine $(200 \mu \mathrm{g})$ and fentanyl $(100 \mu \mathrm{g})$. Concurrently, the fetal heart rate was being rechecked and the parturient was positioned with left lateral uterine displacement, and pre-oxygenated by facemask. UCP was persistent and fetal bradycardia ongoing despite efforts to alleviate cord compression by maintaining cephalad displacement of the fetal presenting part. Induction was achieved with propofol $(200 \mathrm{mg})$ and succinylcholine $(140 \mathrm{mg})$ was administered to facilitate tracheal intubation. The infant was delivered within two minutes after the patient was induced. The Apgar scores were 9 and 9. Following delivery, anesthesia was maintained with sevofluorane in an oxygen and nitrous oxide mixture at 0.5 minimum alveolar concentration (MAC). The patient became hypotensive as the epidural began to take effect (70/30 $\mathrm{mm} \mathrm{Hg}$ ) and was given phenylephrine (300 and 100 phenylephrine (300 and $100 \mu \mathrm{g}$ ) and ephedrine (10 and $5 \mathrm{mg}$ ) to correct the blood pressure. Following the completion of surgical procedures, the patient was awakened and extubated. The patient promptly emerged from anesthesia and her and the infant were in stable condition. She was transferred to the post-anesthesia care unit.

\section{Discussion}

Umbilical cord prolapse is a serious condition, and is one of the few obstetric complications that can transform a routine labour and delivery into a tragic outcome. When a diagnosis of UCP has been made, the risks of maternal anesthetic must be weighed against the urgency of fetal delivery. Options for anesthesia are general or neuraxial (spinal or epidural) anesthesia, and the decision on which to use is dependent on whether there is an epidural catheter already in place and the urgency of fetal delivery. ${ }^{12,13}$

In this case, a decision on whether to proceed with our usual practice of topping up the labour epidural with a concentrated anesthetic appropriate for surgical delivery or to use general anesthesia for a quicker delivery needed to be made rapidly. Upon entry to the OR, the patient was given a top-up of her anesthetic. However, due to the acute fetal distress as evidenced by a persistently low heart rate (80-90 beats per minute), and the fact that the patient had an airway that was expected to allow for uncomplicated intubation (Mallampati Class I), the decision to use general anesthesia in order to expedite the delivery was made.

An emergency cesarean section due to a non-reassuring fetal heart rate is the most common indication for endotracheal intubation during labour and delivery. ${ }^{14}$ The physiologic changes that occur with pregnancy and labour make obstetric patients difficult in regards to airway management, which can result in difficult or failed intubation. The Mallampati score increases throughout the pregnancy as the upper respiratory tract mucosa becomes increasingly vascular and edematous. ${ }^{15}$ Failed intubation is uncommon, the rate of occurrence is $0.4 \%$ in obstetrical patients. ${ }^{16}$ Additionally, the majority of difficult or failed intubations in obstetrics occur during emergencies or outside of regular hours. ${ }^{17}$

In this case the patient's airway examination was reassuring; however, in other circumstances, the therapeutic conflict between fetal versus maternal well-being would have had to been addressed. If the time to delivery of the neonate was prolonged in order to allow for the epidural to take effect, acute neonatal anoxia potentially resulting in brain injury or death could have resulted. However, an attempt to intubate a mother with a difficult airway could potentially result in failed intubation, which can result in severe maternal injury or death. If this were the case, we likely would have resolved the dilemma by adhering to the ethical imperative to put the interests of the mother first.

The patient consented to the publication of this report.

\section{References}

1. Kahana B, Sheiner E, Levy A, Lazer S, Mazor M. Umbilical cord prolapse and perinatal outcomes. Int J Gynaecol Obstet 2004;84(2):127-32.

2. Redline RW. Disorders of placental circulation and the fetal brain. Clin Perinatol 2009;36(3):549-59.

3. Koonings PP, Paul RH, Campbell K. Umbilical cord prolapse: a contemporary look. J Reprod Med 1990;35(7):690-2.

4. Boyle JJ, Katz VL. Umbilical cord prolapse in current obstetric practice. J Reprod Med 2005;50(5):303-6.

5. Gibbons C, O'Herlihy C, Murphy JF. Umbilical cord prolapse: changing patterns and improved outcomes: a retrospective cohort study. BJOG 2014;121(13):1705-8.

6. Dilbaz B, Ozturkoglu E, Dilbaz S, Ozturk N, Sivaslioglu AA, Haberal A. Risk factors and perinatal outcomes associated with umbilical cord prolapse. Arch Gynecol Obstet 2006;274(2):104-7.

7. Behbehani S, Patenaude V, Abenhaim HA. Maternal risk factors and outcomes of umbilical cord prolapse: a population-based study. J Obstet Gynaecol Can 2016;38(1):23-8.

8. Kaymak O, Iskender C, Ibanoglu M, Cavkaytar S, Uygur D, Danisman N. Retrospective evaluation of risk factors and perinatal outcome of umbilical cord prolapse during labor. Eur Rev Med Pharmacol Sci 2015;19(13):2336-9. 
9. Holbrook BD, Phelan ST. Umbilical cord prolapse. Obstet Gynecol Clin North Am 2013;40(1):1-14.

10. Usta IM, Mercer BM, Sibai BM. Current obstetrical practice and umbilical cord prolapse. Am J Perinatol 1999;16(9):47984 .

11. Gabbay-Benziv R, Maman M, Wiznitzer A, Linder N, Yogev Y. Umbilical cord prolapse during delivery-risk factors and pregnancy outcome: a single center experience. J Matern Fetal Neonatal Med 2014;27(1):14-7.

12. McKeen D, George RB, Shukla R. We "can do it" does not mean we "should do it": obesity, umbilical cord prolapse, and spinal anesthesia in the knee-chest position. Can J Anaesth 2009;56(2):168-9.

13. Ginosar Y, Weiniger C, Elchalal U, Davidson E. Emergency cesarean delivery for umbilical cord prolapse: the head-down, knee-chest prone position for spinal anesthesia. Can J Anaesth 2008:55(9):612:61

14. Vasdev GM, Harrison BA, Keegan MT, Burkle CM. Management of the difficult and failed airway in obstetric anesthesia. J Anesth 2008;22(1):38-48.

15. Mushambi MC, Kinsella SM, Popat M, Swales H, Ramaswamy KK, Winton AL, et al. Obstetric Anaesthetists' Association and Difficult Airway Society guidelines for the management of difficult and failed tracheal intubation in obstetrics. Anaesthesia 2015;70(11):1286-306

16. McDonnell NJ, Paech MJ, Clavisi OM, Scott KL, ANZCA Trials Group. Difficult and failed intubation in obstetric anaesthesia: an observational study of airway management and complications associated with general anaesthesia for caesarean section. Int J Obstet Anesth 2008;17(4):292-7.

17. Quinn AC, Milne D, Columb M, Gorton H, Knight M. Failed tracheal intubation in obstetric anaesthesia: 2 yr national case-control study in the UK. Br J Anaesth 2013;110(1):74-80.

\section{WORKSAFE R TRAVAIL SÉCURITAIRE NB}

\section{PHYSICIAN \\ Written and spoken competence in English is required}

WorkSafeNB is accepting applications for a permanent position of physician in Grand Bay-Westfield, New Brunswick.

Reporting to the manager, Work Recovery, the physician will be responsible for conducting history and physical examinations on clients; providing medical diagnosis; prescribing and monitoring medication; and providing appropriate treatment as required, with a focus on the development of a rehabilitation treatment plan and a goal of a safe and healthy return to work. As a member of the Work Recovery team, you will be required to regularly evaluate the clinical progress of clients and to actively participate as member of this multidisciplinary treatment approach. The successful candidate will liaise with the clients' family physicians to facilitate continuity of care for the clients and to provide guidance in the development of treatment plans.

The candidate must be a graduate from an approved medical school, such as family medicine or other appropriate specialties (occupational medicine, physical medicine and rehabilitation, etc.), a licentiate of the Medical Council of Canada, and possess membership in good standing in the College of Physicians and Surgeons of New Brunswick and the New Brunswick Medical Society. The successful candidate must be able to function within MS Office, particularly Word. WorkSafeNB offers an attractive compensation package that includes an equitable salary with excellent benefits, and no weekend or evening work. Please apply in writing to:

\section{Manager, Staffing \\ WorkSafeNB \\ PO Box 160, Saint John NB E2L 3X9 \\ Fax 506 642-0718 or Email employment@ws-ts.nb.ca}

WorkSafeNB reserves the right to raise minimum qualifications depending on the number of applications. WorkSafeNB is an equal opportunity employer and promotes a scent-reduced environment.

WorkSafeNB is committed to preventing workplace injuries and illness through education and the enforcement of the Occupational Health and Safety Act. For more information about WorkSafeNB, or this and other employment opportunities, please visit our website at worksafenb.ca. 